as well as six soluble membrane pro. teins, and have compared them with the compositions of 205 ordinary soluble proteins.

One may argue, of course, about the definition of a polar side chain, but Capaldi and Vanderkooi have taken the simplest view, and have included the charged and the hydroxylic aminoacids as well as glutamine and asparagine. Soluble proteins give a rather narrow distribution of contents of these polar side chains, the great majority lying between 40 and 53 per cent. Only thirteen of the proteins fell below this range. By contrast, the nineteen membrane proteins are evenly spread over a wide range of compositions, nine of them containing less than 40 per cent of polar side chains, five less than 37 per cent. The soluble membrane proteins are all in the range of composition of ordinary globular proteins. One can naturally not generalize about the extent of immersion of proteins in the membrane bilayer, though there is good evidence in one or two instances of proteins projecting right through it.

The dependence of membrane pro. teins on associated lipids for the stability of their functional conformation has been noted in several contexts. A related situation may be that in the serum lipoproteins, which undergo substantial, though reversible, conformational disorganization, when de. nuded of their complement of associated lipids.

An example of the effect on physiological activities of membrane enzymes of the extraction of phopholipids is to be found in the study of Mavis, Bell and Vagelos (J.Biol. Chem., 247, 2835; 1972). One approach, of course, would be to examine activities of isolated enzymes with and without associated phospholipids. Sandermann (FEBS Letts., 21, 254; 1972), for example, has found it possible to reactivate the membrane enzyme, $\mathrm{C}_{55}$-isoprenoid alcohol phosphokinase of Escherichia coli, by adding lecithin. Three different lecithins gave a sharp maximum of activity at a mole ratio of about $800: 1$. The preparation of single membrane proteins in active form often presents insuperable difficulties, however.

The strategy of Mavis et al. is therefore to expose the membranes of $E$. coli to phospholipase C. Unlike phospholipase $A$, which has been used in earlier work, and which leads to the formation of surface active, and therefore potentially denaturing, lysophosphatides, this enzyme produces diglycerides, and phosphoryl compounds, which escape into the solution. The objective then is to relate the destruction of the phospholipids to the activity of various of the enzymes that are known to be lodged in the membrane. The phospholipase $\mathrm{C}$ digests the phospholipids in situ at much the same rate as in the free state, though a fraction of the minor cardiolipin com. ponent resists hydrolysis. Three of the membrane enzymes - species with names of mystic power, that instantly inhibit the thought processes, such as acyl-CoA: glycerol-3-P acyltransferase - - lose activity at the rate at which the phospholipids disappear. The species just mentioned is completely inactivated, and so also is one of the other two. The third suffers a partial inactivation to a limiting level of activity. Two other enzymes, both dehydrogenases, which are associated with the membrane, are unaffected. It seems possible that membrane-embedded enzymes are conformationally dependent on bound phospholipid or on the fluid, paraffin-like nature of their surroundings. Others perhaps are less deeply embedded, or even adhere to the surface.

At the same time, Kung and Henning (Proc. US Nat, Acad. Sci, 69, 925; 1972) report that succinate dehydrogenase, one of the species that is unaffected by digestion of the phospholipids, cannot be extracted from the membrane with a nonionic detergent. Two other dehydrogenases, by contrast, can be extracted and, moreover, in mutants that make them in large excess, are detected by enzymatic assay in the cell supernatant. The inference is that there is a limited number of specific sites on the membrane, which are fully saturated in the mutants. The soluble enzymes apparently take no part in the operation of the respiratory chain. Moreover, the evidence is that several dehydrogenases may compete for the same membrane attachment sites.

A membrane-associated dehydrogenase from red cells has now been isolated by Carraway and Shin (J. Biol. Chem., 247, 2102; 1972). The most interesting feature of their work is that in intact cells this protein, which, as judged by detergent-gel electrophoresis has a subunit molecular weight of some 35,000 , is the only one to be appreciably labelled by radioactive iodoacetate. The reagent is able to penetrate the cell, and therefore has access to a whole range of other proteins; in ghosts other components are in fact attacked, though the glyceraldehyde 3-phosphate dehydrogenase still remains prominent among them. Principally a single cysteine residue is modified.

What is also remarkable is that another thiol reagent, $\mathrm{N}$-ethylimaleimide, shows no such selectivity, and is copiously incorporated into the whole complement of proteins. This shows once again the uncertainty regarding the factors that govern the susceptibility of protein side chains to modifying reagents. It suggests caution in interpreting results such as those of Dratz et al. in Nature New Biology last week (237, $99 ; 1972)$, who report that rhodopsin in intact or fragmented rod membranes, unlike the extracted protein, is not attacked by a reagent with high affinity for amino groups.

\title{
Induced Transplantation Antigen and Malignancy
}

OVER the past several years Harris and Klein and their various colleagues have exploited the technique of cell fusion mediated by inactivated Sendai virus to analyse malignancy. Their studies of the karyotypes and malignancy of hybrid cells obtained by fusing highly malignant cells with cells of low tumorigenicity, and the theories which have stemmed from such experiments, are widely known.

In next Wednesday's issue of Nature New Biology (June 7) Klein and Harris report their latest experiments with such hybrid cells and they conclude that the surface changes which lead in cells transformed by polyoma virus to the appearance of a virus-induced transplantation antigen are not alone sufficient to cause a cell to be malignant in vivo.

Cells transformed by polyoma virus are usually more malignant than their untransformed counterparts, and they also carry a new transplantation antigen which is presumably located at the cell surface and which is certainly virusspecific.

Whether or not this transplantation antigen is coded for by the viral genome, however, remains an open question. Because the polyoma-induced transplantation antigen is exceptionally stable and, for example, survives strong negative selection, it has been suggested in the past by Klein, among others, that the presence of this antigen, or the surface change it reflects, may be an essential requirement for the proliferation in vivo of cells transformed by polyoma virus.

This seems not to be the case, how. ever, for some clones of hybrid cellsproduced by fusing malignant polyoma transformed cells with mouse fibroblasts of low tumorigenicity-prove in inoculation experiments involving syngeneic and semi-allogeneic mice to be of low tumorigenicity even though they retain the polyoma-induced transplantation antigen. Rather the malignancy of such hybrid cells seems to depend on the balance of chromosomes and therefore genetic determinants donated by the malignant and non-malignant parental cells; not until chromosomes from the non-malignant parental cell are lost does the hybrid become malignant. 